\title{
DOUBLY ITERATED MATRIX METHODS OF SUMMABILITY ${ }^{1}$
}

\author{
WILLIAM C. SWIFT
}

1. Introduction. A convenient generalization of the natural operator "limit" is realized in the concept of the summability- $A$ of a sequence with respect to a matrix $A$. For $A=\left(a_{i k}\right)$, the sequence $\left\{x_{k}\right\}$ is said to be summable- $A$ to $x$ provided

$$
x=\lim _{i \rightarrow \infty} \lim _{j \rightarrow \infty} \sum_{k=0}^{j} a_{i k} x_{k} .
$$

Here and throughout, elements of matrices and sequences are to be complex numbers. Indices run from 0 to $\infty$; and in ambiguous cases the sequence index will be repeated as final subscript in the fashion $\left\{c_{n k}\right\}_{n}$.

The matrix $A$ is said to be regular if every convergent sequence is summable- $A$ to its natural limit; for this the requirements on the $a_{i k}$ are the celebrated Silverman-Toeplitz conditions [1, p. 64]. The idea of the present paper derives from the appeal to replace the application of the natural limit in (1) in both instances by summability- $A$ itself, thereby yielding for regular $A$ a not-less-general transform. More generally we consider the succession of functionals defined by repetitions of this double iteration.

Definition 1. With respect to a matrix $A=\left(a_{i k}\right)$, the $A I$-Operator of order $n, W_{n}$, for $n=0,1,2, \cdots$, is the functional, operating on sequences, defined by the following recursion: $W_{0}\left\{x_{k}\right\}=\lim _{k \rightarrow \infty} x_{k}$, and $W_{n+1}\left\{x_{k}\right\}=W_{n}\left\{W_{n}\left\{\sum_{k=0}^{j} a_{i k} x_{k}\right\}_{j}\right\}_{i}$.

Thus $W_{1}\left\{x_{k}\right\}$ is the usual $A$-sum; and by way of example:

$$
W_{2}\left\{x_{k}\right\}=\lim _{p \rightarrow \infty} \sum_{i=0}^{\infty} a_{p i} \lim _{m \rightarrow \infty} \sum_{j=0}^{\infty} a_{m j} \sum_{k=0}^{j} a_{i k} x_{k} .
$$

THEOREM 1. Let $A$ be a regular matrix for which $W_{m}$ and $W_{n}$ are the respective AI-Operators with $m \geqq n$. Then for every sequence $\left\{c_{k}\right\}$ in the domain of definition of $W_{n}$, we have

Presented to the Society, September 1, 1955 under the title $A n$ iterated matrix method of summability; received by the editors November 10, 1960.

1 This paper is based on the author's doctoral dissertation written at the University of Kentucky under the direction of Professor V. F. Cowling, and during the tenure of a Gerard Swope Fellowship awarded by the General Electric Company. 


$$
W_{m}\left\{c_{k}\right\}=W_{n}\left\{c_{k}\right\}
$$

Proof. We consider only the case $m=n+1$; beyond that the proof is evident. For $n=0, m=1$, the conclusion is precisely the condition that $A$ be regular, as hypothesized. Suppose the theorem is valid for $n=p, m=p+1$. To complete the induction, establishing the case $n=p+1, m=p+2$, we must, according to Definition 1 , show that

$$
W_{p+1}\left\{W_{p+1}\left\{\sum_{k=0}^{i} a_{i k} c_{k}\right\}_{j}\right\}_{i}=W_{p}\left\{W_{p}\left\{\sum_{k=0}^{i} a_{i k} c_{k}\right\}_{j}\right\}_{i}
$$

whenever the expression on the right is defined. But from the assumption of the theorem's validity for $n=p, m=p+1$, we have for each $i$,

$$
W_{p+1}\left\{\sum_{k=0}^{j} a_{i k} c_{k}\right\}_{j}=W_{p}\left\{\sum_{k=0}^{j} a_{i k} c_{k}\right\}_{j}
$$

whenever the right side is defined. And similarly for the outside operators.

Definition 2. With respect to regular matrix $A$, a sequence $\left\{c_{k}\right\}$ is said to be summable- $A I_{\omega}$ to $c$, if for $n$ sufficiently large we have $W_{n}\left\{c_{k}\right\}=c$.

NoTE. The relation $\left\{c_{k}\right\}$ summable- $A I_{\omega}$ to $c$ may appropriately be written $W_{\omega}\left\{c_{k}\right\}=c$. Then as in Definition 1 we may define $W_{\omega+1}$; and so on to general ordinal number index. But the problems raised by such generality present a distraction from the classical application that follows and so the subject of transfinite indices is deferred.

In the next section we consider the application of the $A I$-Operators to sequences of functions, extending the idea and useful properties of uniform convergence. In $\$ 3$ we generalize a result of conventional summability- $A$, establishing the effectiveness of the transforms $W_{n}$ for summing Taylor Series in domains larger than the circle of convergence. Finally there is exhibited in $\$ 4$ a simply derived matrix with respect to which the sequence of partial sums of each Taylor Series is summable- $A I_{\omega}$ to its analytic extension throughout the principal star domain of the function.

2. Sequences of functions. Definition 3. With respect to a matrix $A=\left(a_{i k}\right)$, a sequence of functions $\left\{f_{k}(z)\right\}$ is said to be $W_{0}$-uniform for $z$ in a set $T$ if $\left\{f_{k}(z)\right\}$ is uniformly convergent for $z \in T$; and for $n=0,1,2, \cdots,\left\{f_{k}(z)\right\}$ is said to be $W_{n+1}$-uniform for $z \in T$ if for each $i,\left\{\sum_{\mathbf{k}=0}^{j} a_{i n} f_{k}(z)\right\}_{j}$ is $W_{n}$-uniform for $z \in T$, and if in addition $\left\{W_{n}\left\{\sum_{k=0}^{j} a_{i k} f_{k}(z)\right\}_{j}\right\}_{i}$ is defined and $W_{n}$-uniform for $z \in T$. 
It is immediate that " $W_{n}$-uniform" implies " $W_{n}$-summable." For conciseness we combine the concepts, writing simply: " $W_{n}\left\{f_{k}(z)\right\}$ $=f(z)$ uniformly for $z \in T$." It is likewise clear that if $W_{n}\left\{c_{k}\right\}$ is defined, then considering $\left\{c_{k}\right\}$ as a sequence of functions constant over a set $T$ it follows that $\left\{c_{k}\right\}$ is $W_{n}$-uniform for $z \in T$. Here, as in the theorems following, the matrix defining $W_{n}$ is arbitrary, in particular it is not required to be regular.

Theorem 2. Suppose $W_{n}\left\{f_{k}(z)\right\}=f(z)$ and $W_{n}\left\{g_{k}(z)\right\}=g(z)$, both uniformly for $z \in T$, and let $h(z)$ be bounded for $z \in T$. Then

$$
W_{n}\left\{f_{k}(z)+h(z) \cdot g_{k}(z)\right\}=f(z)+h(z) \cdot g(z)
$$

uniformly for $z \in T$.

THEOREM 3. Suppose each element of $\left\{f_{k}(z)\right\}$ is continuous for $z$ in a metric set $T$; suppose also that $\left\{f_{k}(z)\right\}$ is $W_{n}$-uniform for $z \in T$. Then $W_{n}\left\{f_{k}(z)\right\}$ is continuous for $z \in T$.

THEOREM 4. Suppose $\left\{f_{k}(u, z)\right\}$ is $W_{n}$-uniform for $(u, z)$ in a set $C \times T$ where $C$ is a rectifiable contour of the complex plane. Further suppose that for each $k$ and each $z \in T, f_{k}(u, z)$ is continuous for $u \in C$. Then

$$
W_{n}\left\{\int_{C} f_{k}(u, z) d u\right\}=\int_{C} W_{n}\left\{f_{k}(u, z)\right\} d u
$$

uniformly for $z \in T$.

The proofs of these three theorems all conform to the same induction format. In each of them the case $n=0$ is commonplace, and the mechanics of passing from $m$ to $m+1$ is straightforward. We illustrate with the details of the proof of Theorem 4.

Proof of Theorem 4. As just observed the result for the case $n=0$, ordinary uniform convergence, is well known. Assume the theorem valid for $n=m$. Let $\left\{f_{k}(u, z)\right\}$ satisfy the hypotheses for the case $n=m+1$. First observe that

$$
\sum_{k=0}^{j} a_{i k} \int_{C} f_{k}(u, z) d u=\int_{C} \sum_{k=0}^{j} a_{i k} f_{k}(u, z) d u .
$$

From the assumed $W_{m+1}$-uniformity of $\left\{f_{k}(u, z)\right\},\left\{\sum_{k=0}^{j} a_{i k} f_{k}(u, z)\right\}_{j}$ is, for each $i, W_{m}$-uniform for $(u, z) \in C \times T$. The continuity of $\sum_{k=0}^{j} a_{i k} f_{k}(u, z)$ for $u \in C$ follows from the continuity of the respective $f_{k}(u, z)$. Therefore from the $n=m$ case of the theorem we have for each $i$ : 


$$
W_{m}\left\{\int_{C} \sum_{k=0}^{j} a_{i k} f_{k}(u, z) d u\right\}_{j}=\int_{C} W_{m}\left\{\sum_{k=0}^{j} a_{i k} f_{k}(u, z)\right\}_{j} d u
$$

uniformly for $z \in T$.

Again by assumption $\left\{W_{m}\left\{\sum_{k=0}^{j} a_{i k} f_{k}(u, z)\right\}_{j}\right\}_{i}$ is $W_{m}$-uniform for $(u, z) \in C \times T$. For each $i$ and each $z \in T$ the continuity of $W_{m}\left\{\sum_{k=0}^{j} a_{i k} f_{k}(u, z)\right\}_{j}$ follows from Theorem 3 . Thus again from the $n=m$ case of the theorem:

$$
\begin{aligned}
W_{m}\left\{\int_{C} W_{m}\left\{\sum_{k=0}^{j} a_{i \kappa} f_{k}(u, z)\right\}_{j} d u\right\}_{i} & \\
& =\int_{C} W_{m}\left\{W_{m}\left\{\sum_{k=0}^{j} a_{i k} f_{k}(u, z)\right\}_{j}\right\}_{i} d u
\end{aligned}
$$

uniformly for $z \in T$.

Collecting the steps we have

$$
\begin{aligned}
W_{m}\left\{W_{m}\left\{\sum_{k=0}^{j} a_{i k} \int_{C} f_{k}(u, z) d u\right\}_{j}\right\}_{i} & \\
& =\int_{C} W_{m}\left\{W_{m}\left\{\sum_{k=0}^{j} a_{i k} f_{k}(u, z)\right\}_{j}\right\}_{i} d u,
\end{aligned}
$$

the sequences in $j$ and $i$ on the left being $W_{m}$-uniform for $z \in T$. This is precisely the desired $n=m+1$ result.

The linearity of the operators $W_{n}$ we now observe as the all-functions-constant case of Theorem 2. The extension to the following statement is immediate.

Theorem 5. Each AI-Operator $W_{n}$, as well as the summability- $A I_{\omega}$ transform for regular $A$, defines a linear functional over a vector space of sequences of complex numbers.

3. Application to Taylor series. Applying an AI-Operator $W_{n}$ to the partial sums of the Geometric Series, we have:

$$
W_{n}\left\{\sum_{k=0}^{j} z^{k}\right\}_{j}=W_{n}\left\{\frac{1-z^{j+1}}{1-z}\right\}_{j}=\frac{1}{1-z}\left[W_{n}\{1\}_{j}-z W_{n}\left\{z^{j}\right\}_{j}\right]
$$

provided the expression on the right exists. $\left(\{1\}_{j}\right.$ represents the sequence of all 1's). Thus sufficient conditions that $W_{n}$ "properly" sum the Geometric Series at a point $z$ are that $W_{n}\{1\}_{j}=1$ and $W_{n}\left\{z^{j}\right\}_{j}=0$. Theorem 6 provides an analogous result for Taylor Series in general.

A function of the form $f(z)=\sum_{k=0}^{\infty} c_{k} z^{k}$ with positive radius of convergence will be regarded as extended to its principal star domain, 
i.e., if there exists an analytic continuation of $f(z)$ throughout a domain containing the segment $\left\{t z_{0} \mid 0 \leqq t \leqq 1\right\}$ then $f\left(z_{0}\right)$ represents the value defined thereby. We represent the principal star domain as $M_{f}$. Note that its complement $\mathrm{e} M_{f}$ consists of those points which are singularities of the analytic function $f(z)$ by a radial approach, automatically including all points "in the shadow of" such singularities.

In general a region $Q$ is said to be starlike if for each $z \in Q$ we have $t z \in Q$ for $0 \leqq t \leqq 1$. For a starlike domain $Q$, the partial star domain $P_{f Q}$ of $f(z)$ with respect to $Q$ is the intersection of the sets $\zeta Q$ as $\zeta$ ranges over $\mathfrak{e} M_{f}$. $(\zeta Q$ represents the set $\{\zeta z \mid z \in Q\})$.

The following result is an extension of a theorem of Okada [1, p. 189] applying for conventional matrix summability.

THEOREM 6. Let $f(z)=\sum_{k=0}^{\infty} c_{k} z^{k}$ have positive radius of convergence, and let $s_{j}(z)=\sum_{k=0}^{j} c_{k} z^{k}$. Let $W_{n}$ be an AI-Operator with the properties that $W_{n}\{1\}_{k}=1$ and $W_{n}\left\{z^{k}\right\}_{k}=0$ uniformly for $z$ in each closed and bounded set in a starlike domain $Q$. Then $W_{n}\left\{s_{j}(z)\right\}=f(z)$ uniformly for $z$ in each closed and bounded set in the partial star domain $P_{f Q}$ of $f(z)$ with respect to $Q$.

Proof. Since $Q$ clearly cannot contain the point $z=1$ it follows that $P_{f Q}$ is a subset of $M_{f}$. And since otherwise the theorem is vacuous we assume that $Q$ contains the origin.

Let $T$ represent a closed and bounded set in $P_{f Q}$. For $j=0,1,2, \cdots$, consider the integral:

$$
I_{j}(z)=\frac{1}{2 \pi i} \int_{C} \frac{f(u)}{z-u}\left(\frac{z}{u}\right)^{j+1} d u .
$$

Here $C$ is a rectifiable simple closed curve, taken counterclockwise, with the properties: $f(z)$ is analytic on and inside $C$; the origin and the set $T$ are properly inside $C$; and the union of all points of the form $t / u$ for $t \in T$ and $u \in C$ forms a closed and bounded subset of $Q$.

To verify the existence of such a contour $C$ without a tedious direct construction we note that since $M_{f}$ clearly satisfies the hypotheses of the Riemann Mapping Theorem there is an analytic function $g(z)$ which simply maps the unit circle $|z|<1$ onto $M_{f}$. Since $T$ is a closed and bounded set in $M_{f}$ the pre-image of $T$ under this mapping will be contained in a circle $|z|<\rho_{1}<1$. Furthermore the definition of $P_{f Q}$ insures that for all $t \in T \subset P_{f Q}$ and $\zeta \in \mathbb{C} M_{f}$ we have $(t / \zeta) \in Q$. Thus if $\gamma \in \mathbb{e} Q$ it follows that $(t / \gamma) \in M_{f}$. Let $S$ represent the union of the origin and all points of the form $t / \gamma$ as $t$ ranges over the closed and bounded set $T$ and $\gamma$ ranges over the closed and bounded-away-fromzero set $\mathrm{e} Q$. Clearly $S$ is a closed and bounded set in $M_{f}$. As with $T$, 
the image of $S$ under the inverse mapping $g^{-1}(z)$ lies in a circle $|z|<\rho_{2}<1$. To recapitulate: $t \in T$ implies $\left|g^{-1}(t)\right|<\rho_{1}<1$; and $t \in T$ and $\rho_{2} \leqq|z|<1$ implies $(t / g(z)) \in Q$. It follows immediately that the image under $g(z)$ of the circle $|z|=\max \left(\rho_{1}, \rho_{2}\right)$ furnishes an acceptable contour $C$.

The integrand in (2) is analytic inside $C$ except for poles at $u=0$ and $u=z$. Observing that $I_{j}(0)=0$ and $f(0)=s_{j}(0)=c_{0}$, we pass on to the case $z \neq 0$. The residue at $u=z$ is clearly $-f(z)$. Near $u=0$ the integrand may be expanded thus:

$$
\begin{aligned}
\frac{z^{j+1}}{u^{j+1}} \cdot \frac{1}{z}\left(c_{0}+c_{1} u+\cdots+c_{k} u^{k}+\cdots\right) & \\
& \cdot\left(1+\frac{u}{z}+\cdots+\frac{u^{k}}{z^{k}}+\cdots\right) .
\end{aligned}
$$

Collecting the coefficient of $u^{-1}$ we have the residue:

$$
z^{j}\left(\frac{c_{0}}{z^{j}}+\frac{c_{1}}{z^{j-1}}+\cdots+c_{j}\right)=s_{j}(z) .
$$

It follows therefore that for all $z \in T$,

$$
I_{j}(z)=s_{j}(z)-f(z) .
$$

From the hypotheses of the theorem, Theorems 2 and 4, and the properties of $C$, it is clear that

$$
W_{n}\left\{I_{j}(z)\right\}_{j}=\frac{1}{2 \pi i} \int_{C} \frac{f(u)}{z-u} \cdot \frac{z}{u} \cdot W_{n}\left\{\left(\frac{z}{u}\right)^{j}\right\}_{j} d u=0
$$

uniformly for $z \in T$. Recalling Theorem 2 and the fact that $W_{n}\{1\}_{k}$ $=1$, we have finally

$$
W_{n}\left\{I_{j}(z)+f(z)\right\}_{j}=f(z)
$$

uniformly for $z \in T$. Since

$$
I_{j}(z)+f(z)=s_{j}(z)
$$

this is the desired result.

4. An example. Henceforth let $G(z)=(2-z)^{-1}$, and let $A=\left(a_{i k}\right)$ be the matrix whose elements are the coefficients of the Taylor Series of the functions $[G(z)]$. To be precise

$$
[G(z)]^{i}=(2-z)^{-i}=\sum_{k=0}^{\infty} a_{i k} z^{k} \quad \text { for }|z|<2 .
$$

Hence 


$$
a_{i k}=(1 / 2)^{i+k} C_{i+k-1, i-1} .
$$

This matrix $A$, or more specifically the summability- $A$ functional associated with it, represents the $\alpha=1 / 2$ case of the $S_{\alpha}$ methods which correspond to the family of matrices defined in the manner of (3) from the functions $G_{\alpha}(z)=(1-\alpha) /(1-\alpha z)$ for $0<\alpha<1$ [2]. That $A$ is regular follows immediately from the Silverman-Toeplitz Conditions. However we note that it is an easy exercise to demonstrate by an $(\epsilon, N)$-type argument that a given convergent sequence is summable$A$ to its natural limit. The rest of the paper is devoted to demonstrating the following result.

TheOREM 7. Let $W_{n}$ be the AI-Operator of order $n$ for the matrix $A$ given by (4). Let $f(z)=\sum_{k=0}^{\infty} c_{k} z^{k}$ have positive radius of convergence, and let $s_{j}(z)=\sum_{k=0}^{j} c_{k} z^{k}$. Then for each closed and bounded set $T$ in the principal star domain $M_{f}$ of $f(z)$, there is an $N$ such that for all $n>N$,

$$
W_{n}\left\{s_{j}(z)\right\}_{j}=f(z)
$$

uniformly for $z \in T$.

For a set $T$ consisting of a single point, Theorem 7 takes the form:

Corollary. Let $A, f(z)$ and $s_{j}(z)$ be as in Theorem 7. Then $\left\{s_{j}(z)\right\}$ is summable-A $I_{\omega}$ to $f(z)$ throughout the principal star domain of $f(z)$.

In point of fact there have been exhibited matrices with respect to which the usual summability method (our $W_{1}$ ) sums $\left\{s_{j}(z)\right\}$ to $f(z)$ throughout $M_{f}[1$, pp. 181-187]. But the construction of our matrix is very much simpler, and although summability- $A I_{\omega}$ arithmetically transcends ordinary summability- $A$ it is nonetheless a perfectly natural generalization. Moreover the proof of Theorem 7 requires no more that an application on Theorem 6 together with a straightforward geometrical argument. The argument may clearly be adapted to a general class of matrices; in particular we note that Theorem 7 may be established in similar fashion for the matrices of all $S_{\alpha}$ methods where $1 / 2 \leqq \alpha<1$.

Proof of Theorem 7. Since the matrix $A$ is regular it follows from Theorem 1 that $W_{n}\{1\}_{k}=1$ for all $n$. Suppose for given $n, W_{n}\left\{z^{k}\right\}_{k}=0$ uniformly in each closed set in a bounded starlike domain $R_{n}$. Then applying Theorem 6 it follows that $W_{n}\left\{\sum_{k=0}^{j} a_{i k} z^{k}\right\}_{j}=[G(z)]^{i}$ for $z \in 2 R_{n}$, and $W_{n}\left\{[G(z)]^{i}\right\}_{i}=0$ for $z \in G^{-1}\left(R_{n}\right)$, both with the usual uniformity in closed subsets. (Here, $2 R_{n}=\left\{z \mid(z / 2) \in R_{n}\right\}$ and $G^{-1}\left(R_{n}\right)$ $=\left\{z \mid G(z) \in R_{n}\right\}$.) Thus if $R_{n+1}$ is a bounded starlike domain contained in $G^{-1}\left(R_{n}\right) \cap 2 R_{n}$ then $W_{n+1}\left\{z^{k}\right\}_{k}=0$ uniformly in each closed set in $R_{n+1}$. 
With $G^{0}(z)=z$ and $G^{n+1}(z)=G\left(G^{n}(z)\right)$, let $D_{n}$ represent the domain in which $\left|G^{n}(z)\right|<1$. Thus $D_{0}$ is the unit circle; and with the usual linear fractional transformation manipulations it follows inductively that for $n=1,2, \cdots, D_{n}$ is the domain defined by the following inequality:

$$
D_{n}:\left|z-\left(1+\frac{1}{2 n-1}\right)\right|>\frac{1}{2 n-1} .
$$

Let $D_{n}^{*}$ represent the starlike part of $D_{n}$, i.e., $D_{n}^{*}$ consists of those points $z$ of $D_{n}$ for which the segment $\{t z \mid 0 \leqq t \leqq 1\}$ does not intersect the circle bounding $D_{n}$. Finally let

$$
Q_{n}=\bigcap_{k=0}^{n} 2^{k} D_{n-k}^{*} .
$$

From the definition it follows that $Q_{n}$ is bounded and starlike, that $Q_{n+1}=D_{n+1}^{*} \cap 2 Q_{n}$ and that $Q_{n+1} \supset Q_{n}$ with $\cup_{n=0}^{\infty} Q_{n}$ being precisely the complement of the segment $\{x \mid 1 \leqq x\}$.

Lemma. With $W_{n}$ defined as in Theorem $7, W_{n}\left\{z^{k}\right\}_{k}=0$ uniformly for $z$ in each closed set in the domain $Q_{n}$ of (6).

Proof of Lemma. Since $Q_{0}$ is the unit circle, the $n=0$ (natural convergence) case of the lemma is immediate. To deduce the validity of the lemma for $n+1$ assuming its validity for $n$, we employ the argument spelled out above in terms of domains $R_{n}$ and $R_{n+1}$. That $Q_{n}$ is bounded and starlike has already been observed, so we have only to demonstrare that $Q_{n+1} \subset G^{-1}\left(Q_{n}\right) \cap 2 Q_{n}$. But as has also been observed, $Q_{n+1}=D_{n+1}^{*} \cap 2 Q_{n}$, so it suffices to show that $D_{n+1}^{*} \subset G^{-1}\left(Q_{n}\right)$. Referring to (6) the proof reduces to showing that $D_{n+1}^{*} \subset G^{-1}\left(2^{k} D_{n-\mathbf{k}}^{*}\right)$ for $k=0,1, \cdots, n$.

The character of the domains $D_{n+1}^{*}, 2^{k} D_{n-k}^{*}$ and $G^{-1}\left(2^{k} D_{n-k}^{*}\right)$, for $k=0,1, \cdots, n-1$, is indicated in Figure 1 . The former two are plotted directly from definition; the construction of $G^{-1}\left(2^{k} D_{n-k}^{*}\right)$ for the transformation $G^{-1}(z)=2-z^{-1}$ is immediate. From the geometry it is clear that the condition $D_{n+1}^{*} \subset G^{-1}\left(2^{k} D_{n-k}^{*}\right)$ is equivalent to the following inequality comparing the ratio of diameter to distance from the origin for the circles generating $D_{n+1}^{*}$ and $G^{-1}\left(2^{k} D_{n-k}^{*}\right)$ respectively:

$$
\begin{aligned}
\frac{2}{2 n+1} \geqq \frac{1}{2-2^{-k}}[ & \left(2-\left(2^{k}\left(1+\frac{2}{2(n-k)-1}\right)\right)^{-1}\right) \\
& \left.-\left(2-2^{-k}\right)\right] .
\end{aligned}
$$



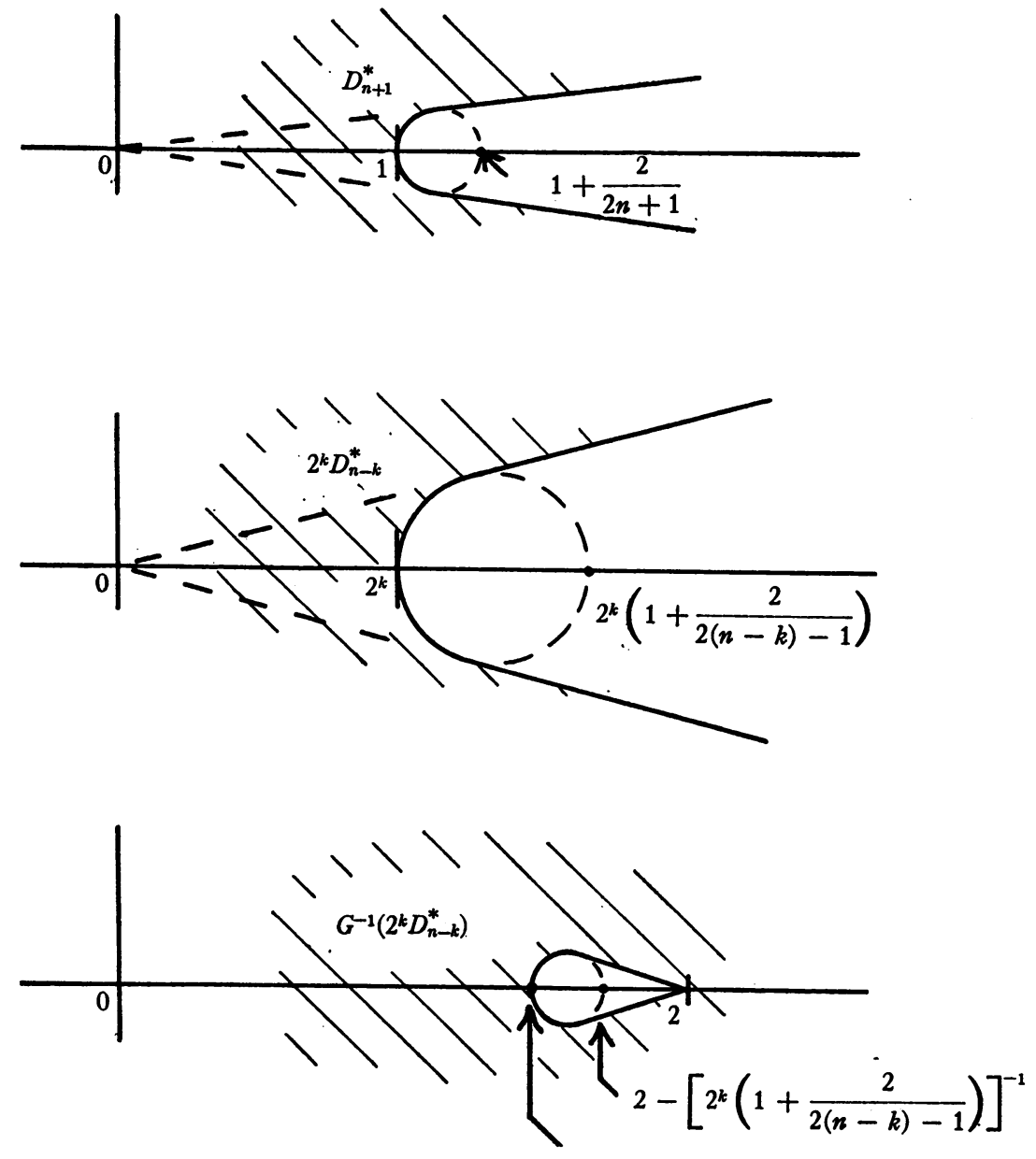

$1 \leqq 2-2^{-k}$

Figure 1

In the exceptional case $k=n$, the region $2^{k} D_{n-k}^{*}$ is simply the circle $|z|<2^{n}$, and $G^{-1}\left(2^{k} D\right)_{n-k}^{*}$ is the domain $|z-2|>2^{-n}$. Reasoning as before we find that the condition $D_{n+1}^{*} \subset G^{-1}\left(2^{k} D_{n-k}^{*}\right)$ for $k=n$ is indeed equivalent to the $k=n$ case of the inequality (7). Thus the proof of the lemma has reduced to proving the inequality (7) for $n=0,1,2, \cdots$ with $k=0,1, \cdots, n$. Simplifying (7), we obtain the following equivalent forms: 


$$
\begin{aligned}
& \frac{2}{2 n+1} \geqq \frac{1}{2^{k+1}-1}\left[1-\frac{2(n-k)-1}{2(n-k)+1}\right]=\frac{2}{\left(2^{k+1}-1\right)(2(n-k)+1)}, \\
& \left(2^{k+1}-1\right)(2(n-k)+1) \geqq 2 n+1, \\
& \left(2^{k+1}-2\right) \cdot 2(n-k)+\left(2^{k+1}-2 k-2\right) \geqq 0 .
\end{aligned}
$$

Since in the last form both terms on the left are clearly non-negative for admissible values of $n$ and $k$, the desired inequality is established. This completes the proof of the lemma.

Returning to the proof of the theorem proper, recall that for the domains $Q_{n}$ of (6), $Q_{n+1} \supset Q_{n}$ and $\cup_{n=0}^{\infty} Q_{n}$ omits only the half-line $[1, \infty]$. Since the principal star domain $M_{f}$ is starlike, $z \in M_{f}$ and $\zeta \in \mathcal{C} M_{f}$ implies $(z / \zeta) \notin[1, \infty]$. Thus for a closed and bounded set $T$ contained in $M_{f}$, the union of the origin and all points of the form $z / \zeta$ for $z \in T$ and $\zeta \in \mathbb{C} M_{f}$ forms a closed and bounded set $V$ not intersecting $[1, \infty]$. Clearly for $n$ sufficiently large, $V \subset Q_{n}$. But for $z \in T: \zeta \in \mathbb{e} M_{f}$ implies $(z / \zeta) \in V \subset Q_{n}$, or in other words, $z \in \zeta Q_{n}$; and thus $z \in P_{f Q_{n}}$. Therefore $T$ is a closed and bounded set in $P_{f Q_{n}}$. Recalling the lemma and the fact that $W_{n}\{1\}_{k}=1$, and applying Theorem 6 , the conclusion of Theorem 7 follows.

\section{REFERENCES}

1. R. G. Cooke, Infinite matrices and sequence spaces, Macmillan and Co., Ltd., London, 1950.

2. W. Meyer-König, Untersuchungen über einige verwandte Limitierungsverfahren, Math. Z. vol. 52 (1949) pp. 257-304.

UNIVERSITY OF KENTUCKY AND

RUtGers, the State University 